\title{
Immediate sensorimotor grounding of novel concepts learned from language alone
}

\author{
Fritz Günther ${ }^{1}$, Tri Nguyen ${ }^{2}$, Lu Chen $^{3}$, Carolin Dudschig ${ }^{1}$, Barbara Kaup ${ }^{1}$, and Arthur M. Glenberg $2,4,5$ \\ ${ }^{1}$ Department of Psychology, University of Tübingen, Tübingen, Germany \\ ${ }^{2}$ Department of Psychology, Arizona State University, Tempe, AZ, United States \\ ${ }^{3}$ Department of English, Shanghai International Studies University, Shanghai, China \\ ${ }^{4}$ INICO, University of Salamanca, Salamanca, Spain \\ ${ }^{5}$ Department of Psychology, University of Wisconsin-Madison, Madison, WI, United States
} Note: This is the author's preprint version of the article (date: 17. 08.2020$)$. The final
article is accepted for publication in the Journal of Memory and Language.

Theories of grounded cognition postulate that concepts are grounded in sensorimotor experience. But how can that be for concepts like Atlantis for which we do not have that experience? We claim that such concepts obtain their sensorimotor grounding indirectly, via already-known concepts used to describe them. Participants learned novel words referring to up or down concepts (mende $=$ enhanced head or mende $=$ bionic foot). In a first experiment, participants then judged the sensibility of sentences implying up or down actions (e.g., "You scratch your bionic foot") by performing up or down hand movements. Reactions were faster when the hand movement matched the direction of the implied movement. In the second experiment, we observed the same congruency effect for sentences like, "You scratch your mende", whose implied direction depended entirely on the learning phase. This offers a perspective on how concepts learned without direct experience can nonetheless be grounded in sensorimotor experience.

Keywords: Grounded cognition; embodiment; word learning; language comprehension

If a traveller told a medieval English farmer that a zebra is a horse with stripes, and that he rode one during his travels through foreign lands, she would probably be amazed, but her cognitive system would be ready and fully able to deal with this information - even if it involves a

This work was supported by Research Fellowship 392225719 from the German Research Foundation (DFG) awarded to Fritz Günther and by the DFG Collaborative Research Center SFB 833: The construction of meaning, Project Z2, appointed to Barbara Kaup. Lu Chen was supported by grant 201706905009 from the China Scholarship Council. This research (STUDY00007649) was determined to be "exempt" by the Arizona State University IRB. All data and analysis scripts are available at the Open Science Framework (https://doi.org/10.17605/OSF.I0/FTXJY). We wish to thank all our student assistants for their great help in the data collection. We also wish to thank Victor Kuperman and two anonymous reviewers for their helpful feedback on earlier versions of this manuscript. so-called "zebra" which she has never even heard of, let alone seen before (for a graphical illustration, see

https://io9.gizmodo. com/how-europeans-imagined-exotic-animals -centuries-ago-ba-1545362205). This scenario poses a serious challenge for theories of grounded cognition (Barsalou, 2008; Fischer, 2012), which assume that the conceptual system and semantic memory need to be grounded in sensorimotor experience with the outside world (e.g. Barsalou, 1999; Glenberg \& Kaschak, 2002; Harnad, 1990; Zwaan \& Madden, 2005). While the exact definition of grounded is debated in the literature, in the present article we adapt a minimalist working definition: A concept is grounded if it is connected to sensorimotor experience (compare Mahon, 2015). Even with this minimalist definition, the question very much remains: How can the farmer ground "zebra" in our story? If this very simple question remains unanswered, claims that the conceptual system is or needs to be grounded cannot be sustained.

In the present article, we suggest an answer to this question and test it: indirect grounding. That is, novel words 
describing novel concepts can be grounded by extrapolating from the sensorimotor experience connected to the alreadyknown words of the definition. We first propose a mechanism of how indirect grounding can be established, before introducing an experimental test for it.

According to the experiential trace model (Zwaan \& Madden, 2005), grounding is established through the systematic co-occurrence between linguistic stimuli (e.g., hearing the word horse) and sensorimotor experience (e.g., seeing or riding a horse) - with co-occurrences between stimuli being a main organizing principle of our conceptual system (Günther, Rinaldi, \& Marelli, 2019; Landauer \& Dumais, 1997). During processing, the linguistic stimulus then acts as a cue that re-activates the sensorimotor experience. According to some accounts of grounded cognition, this re-activation enables the speaker to understand the concept (however, this ascribed causal role for comprehension is strongly debated; see Fischer \& Zwaan, 2008; Strozyk, Dudschig, \& Kaup, 2019; and the General Discussion of the present article). In a series of studies, Lachmair, Dudschig, De Filippis, de la Vega, and Kaup (2011) provide empirical evidence for the cued re-activation postulated by the experiential trace model: They presented participants with words associated to either an upper or lower vertical location (sun versus basement). When performing tasks that do not require lexical access to words (and for which, by implication, access to word meanings is fully task-irrelevant), such as vertical response movements towards the stimuli's font colour, participants were faster when the movement direction matched the associated vertical location (a congruency effect; see also Thornton, Loetscher, Yates, \& Nicholls, 2013, for converging results). The same results were observed for novel words whose referents where just physically encountered in specific vertical locations in a learning phase before the actual experiment (Öttl, Dudschig, \& Kaup, 2017), demonstrating directly that sensorimotor experiential traces formed during interaction with these referents are automatically re-activated during language processing.

However, as highlighted at the beginning of this article, there is a serious limitation to these results: We acquire new semantic representations on a regular basis, often without any accompanying change in our perceptual environment or sensorimotor experience. Landauer and Dumais (1997) estimate that high school students learn the meaning of 1015 new words each day, and to a somewhat lesser extent, this continues throughout our lifetime (Brysbaert, Stevens, Mandera, \& Keuleers, 2016). Thus, new words and concepts are often acquired from language alone. This poses a serious challenge for the scalability of grounded theories of cognition - how can we re-activate sensorimotor experience with a referent if we never had any such experience?

This challenge has long been recognized for abstract words such as freedom (see Borghi et al., 2017;
Pecher, 2018). Prominent possible grounding mechanisms for abstract words discussed in the literature include metaphorical grounding, for example grounding time via space (Lakoff \& Johnson, 1980), grounding abstract concepts in emotions (Kousta, Vigliocco, Vinson, Andrews, \& Del Campo, 2011; Vigliocco et al., 2013), or in their use as social tools and therefore an experience in their own right (Borghi \& Binkofski, 2014). However, the underlying problem clearly goes beyond a simple concrete-abstract distinction: How can even concrete concepts such as atom, Atlantis, or supernova be grounded in experience? In the context of such non-experienced concrete concepts, focussing on the special status of abstract words may fall short of a comprehensive account.

However, a possible solution is already offered in the work that sparked the grounded cognition debate in the cognitive sciences (Harnad, 1990): Zebra can be easily understood by our medieval European if she knows the meaning of horse and stripe, and is told by the traveller that a zebra is a horse with stripes. In terms of the experiential trace model, zebra would be grounded indirectly, through linguistic co-occurrence with already-grounded concepts - in this case, through being described in terms of already-grounded concepts and therefore incorporating these experiential traces (compare Snefjella, Lana, \& Kuperman, in press; see also Snefjella \& Kuperman, 2016 for earlier versions of the same argument). Based on this assumption, Günther, Dudschig, and Kaup (2018) investigated whether novel words that were learned from language alone can lead to sensorimotor activation during language processing. In five high-powered experiments, participants performed the same test phase as in Öttl et al. (2017), after learning the novel words in different, purely language-based learning paradigms that associated these words with a vertical orientation. Contrary to predictions based on indirect grounding, no congruency effects were observed.

However, given the non-semantic nature of the color-judgment task, these results only show that sensorimotor experiential traces are not automatically activated whenever a word is encountered (Ostarek \& Huettig, 2019). Indeed, even activation of the most prominent sensorimotor experience is highly context-dependent (Lebois, WilsonMendenhall, \& Barsalou, 2015). Thus, these results do not conclusively demonstrate that these concepts are not connected to sensorimotor experience (i.e., not grounded). In the present study, we address this issue by moving from a paradigm that is designed to detect low-level automatic activation (and does not even require lexical access) to a highlevel semantic task: reading for comprehension (compare Fischer \& Zwaan, 2008). If even in this task - which necessarily requires not only lexical, but full semantic access we find no evidence for the grounding of newly-learned con- 
cepts, claims about the grounding of the conceptual system would be rendered untenable.

For familiar concepts, previous studies report evidence for the activation of sensorimotor experience during comprehension: In a study by Glenberg and Kaschak (2002), participants read sentences such as You open the drawer or You close the drawer, and judged the sentence sensibility with hand movements towards or away from their body. Responses were faster when the direction of the hand movement matched direction of the described movement. This effect was also observed in the vertical dimension, for sentences such as You touch your ankle (Borghi, Glenberg, \& Kaschak, 2004). Note, however, that the literature also reports failures to find a congruency effect in some versions of the Glenberg and Kaschak task (e.g. Papesh, 2015). Thus, in the present experiment, we use a version of the task that has been frequently replicated (Dudschig \& Kaup, 2017; Lachmair et al., 2011; Öttl et al., 2017; Thornton et al., 2013), and base our experiments on a power analysis to establish the adequacy of our method and sufficiency of our sample size.

Here, we test whether congruency effects also occur during the processing of sentences describing an action with novel concepts learned from language alone. Participants first learn slightly futuristic upwards-associated concepts (for example, mende = enhanced head: a head in which a thin net of electrodes is implanted) and downwards-associated concepts (seige = bionic foot). In the first experiment, they then use up and down hand movements to judge the sensibility of sentences implying up or down actions (You scratch your enhanced head or You scratch your bionic foot). A congruency effect in this first experiment would provide further evidence that sensorimotor experience is activated during the processing of sentences describing vertical actions (in line with Borghi et al., 2004), even when the described actions involve novel concepts. This is especially important given previous non-replications of similar effects (Papesh, 2015); therefore, the presence of such a congruency effect would render our experimental paradigm adequate to investigate our main research question.

The critical second experiment is then set up to decisively test whether novel concepts, whose novel labels are learned from language alone, can be indirectly grounded in sensorimotor experience. In this second experiment, participants judge the sensibility of sentences such as You scratch your mende. Crucially, the implied direction and sensibility of these sentences depend entirely on the completely counter-balanced and randomized name-to-concept assignments in the learning phase. A congruency effect in this second experiment would be in line with the notion of sensorimotor activation, even if no sensorimotor experience has ever been made with a word's referent. This would imply that the word meanings can be grounded indirectly, and ensure that any congruency effects in the first experiment is not a surface-level effect caused by the presence of words such as head and foot in the sentence. It would also suggest an actual motor simulation of the described action (Glenberg $\&$ Kaschak, 2002), rather than just a re-enactment or the reactivation of past experience: The action itself (scratching) does not imply a vertical movement. It only does so when it involves certain objects, but since these objects are novel concepts, the specific actions described in the sentences have never been performed before.

\section{Experiment 1}

\section{Method}

Participants. Adapting the sample size determined by Günther et al. (2018), we tested 49 native German speakers (9 identified as male, 40 as female, $M_{\text {Age }}=23.4$ years, $S D_{\text {Age }}=3.8$ years). One additional participant was not included in the analysis due to a high error rate (see the Results section). In all experiments reported in this article, participants gave informed consent to participating in the experiment, and received either money or course credit for their participation.

Material. The materials for this experiment are based on eight different concepts. Four of the concepts are created by modifying words referring to objects typically located in upper locations (screen ceiling, organic smoke detector, color-changing hair, enhanced head). The other four concepts are created by modifying words referring to objects typically located in lower locations (grass carpet, crystal floor, bionic foot, massage sock). ${ }^{1}$ Each novel concept had a two-sentence description explaining it in more detail (see Table 1). None of the concepts had an associated word in German. In a survey with an independent sample of 35 participants, the vast majority of participants indicated that they had never heard of these concepts before, and those who had overwhelmingly indicated that they had no direct experience with them (see Figure 1). ${ }^{2}$ For ease of reference, we will therefore still refer to these "novel word meanings that in most cases coincide with novel, non-experienced concepts for the individual participants" simply as "novel concepts".

Using these novel concepts, we constructed 40 sentences (five per concept) of the format You VERB your NOVEL CONCEPT. All of the sentences including up-words

\footnotetext{
${ }^{1}$ All the actual experimental material was in German; we use English translations here for illustration.

${ }^{2}$ The pattern of results for all experiments reported in the article stay the same when excluding the item grass carpet, which appears to be more familiar to some people.
} 


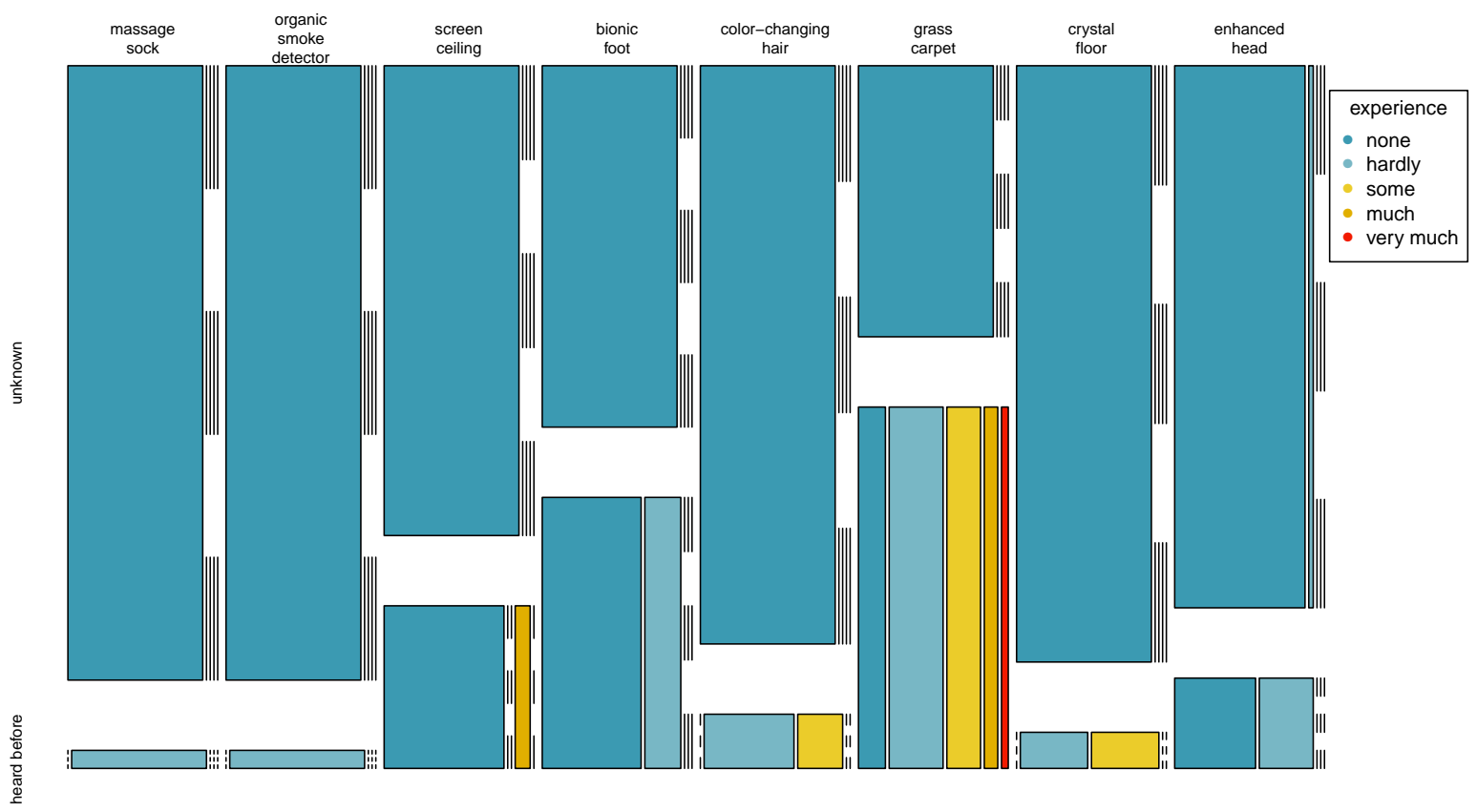

Figure 1. Participant ratings on whether they have heard of the novel concepts before, and if so, how much experience they had made with them.

described plausible upwards hand movements, and all the sentences including down-words described plausible downwards hand movements. We took care that the 23 verbs (e.g., scratch, massage, and operate) did not imply any vertical movements on their own. A sizeable portion of sentences were implausible if the novel concept was replaced by its unmodified noun (You disassemble your [bionic] foot or You switch off your [screen] ceiling). Using the same pool of novel concepts and verbs, we further created a total of 40 sentences (five per concept) describing implausible actions (You massage your screen ceiling). In the final material consisting of 80 sentences, each verb occurred at least once in one plausible and one implausible sentence, and at least in one sentence describing an upwards movement and one describing a downwards movement. Each verb was used in two to six different sentences.

Additionally, we employed eight different Germansounding novel words (Fente, Essede, Seige, Mende, Emahte, Greites, Gehaff, Riehrer) that were already used in the experiments by Günther et al. (2018) and were not a priori associated to a vertical dimension. We constructed eight different learning items for each participant by randomly pairing these novel words with the eight novel concepts and their short descriptions (see Table 1 for examples).

\section{Procedure.}

Learning Phase. Participants were first instructed to learn the novel word meanings, and then presented with the eight different learning items in a randomized order. Following this initial presentation, the eight novel words were presented in random order, and participants were asked to type in the corresponding novel concept for each pseudoword (e.g., enhanced head, not the two-sentence description). Participants received feedback for their answers, and if they typed in an incorrect answer, the respective learning item was immediately presented again. After all eight novel words were presented, participants received feedback on their total number of errors, and were instructed that they would be tested on the novel word meanings once again. This procedure was repeated until all items were answered correctly two times in a row. Once an item was answered correctly two times in a row, it was no longer presented to the participants.

Note that, in Experiment 1, the correct learning of novel words is not directly relevant for the test phase, but it was required here to ensure maximal comparability to Experiment 2 . In addition, assigning a label can also be expected to reinforce the representation of the novel concepts as distinct 
Table 1

Examples for Learning Phase items

$$
\text { mende }=\text { enhanced head }
$$

A mende is a head in which a very thin net of electrodes is implanted. People with a mende can interact directly with electronic devices, without the need for additional input devices like keyboards.

$$
\text { seige }=\text { bionic foot }
$$

A seige is a biomechanical foot that was initially developed to replace amputated feet, and is equipped with high-performing mechanical muscles. For its user, it looks and feels exactly like a real foot.

entities.

Test Phase. After completing the learning phase, participants sat in front of the computer monitor and a vertically mounted keyboard with a special four-button overlay (upper- and lower-middle buttons, one upper button and one lower button, see Figure 2).

Participants started each trial by holding down the two middle buttons of the keyboard. Pressing the buttons initiated presentation of a white fixation cross on the black background in the center of the screen for $1000 \mathrm{~ms}$. If participants released one of the middle keys during this time, they were instructed to press it again. Then, one of the sentences appeared in the center of the screen in white letters on black background. Participants were instructed to judge whether the sentences presented to them described a sensible action or not. Half of the participants were instructed to release the upper middle button and to press the upper button when judging a sentence as sensible, and the other half was instructed to release the lower middle button and press the lower button (vice versa for nonsensical sentences). All responses to sensible sentences were to be made with the dominant hand. The time it took to release a middle button was recorded as reaction time (Günther et al., 2018; Lachmair et al., 2011; Öttl et al., 2017). If their response was correct, they received a Correct! feedback in green letters for $1000 \mathrm{~ms}$. If participants released the wrong middle button or pressed the wrong outer button, they received an Error! feedback for $1500 \mathrm{~ms}$ in red letters. At the end of each trial, a blank black screen was presented for $500 \mathrm{~ms}$ before a new trial started.

The 80 sentences were presented in one experimental block in random order, with the restriction that the same novel concept did not appear twice within a window of four consecutive items. The experimental block was preceded by a training block consisting of ten sentences (five plausible, five implausible) that did not describe vertical movements. None of the nouns used in the experimental material appeared in the training block.

After finishing the first experimental block, participants were informed that the response assignment would now be reversed: Participants who previously reacted towards plau- sible sentences with upwards movements would now do so with downwards movements, and vice versa. After this instruction, participants were again presented with the practise block, followed by the second experimental block.

\section{Results}

Practice trials and nonsense items were excluded from the data set. We further eliminated the data from one participant with high error rates (defined as $<70 \%$ correct for either sensible or nonsensical items) and two high-error items (correct answers not significantly above chance level, $<65$ $\%$ correct). Then, all error trials (11.3\% of trials) were omitted from the data. We further excluded as absolute outliers trials with extremely high and extremely low overall reaction times (faster than the .01-quantile, $780 \mathrm{~ms}$, or slower than the .98-quantile, $3857 \mathrm{~ms}$ ), and as relative outliers trials that deviated more than 2.5 SDs from the mean value per participant per condition ( $1.7 \%$ of trials). ${ }^{3}$

Using these data, we estimated Linear Mixed Effect Models (LMEMs Baayen, Davidson, \& Bates, 2008) using the R packages lme4 (Bates, Mächler, Bolker, \& Walker, 2015) and lmerTest (Kuznetsova, Brockhoff, \& Christensen, 2017). Our model to predict log-transformed release times (Baayen \& Milin, 2010) included a fixed effect for congruency, random intercepts for participants and items, and byparticipant random slopes for congruency. 4

This model including the congruency fixed effect significantly outperformed a model without this effect in a likelihood-ratio test $\left(\chi^{2}(1)=10.41, p=.001, b=0.03\right.$, $t=3.23$ ), indicating a significant congruency effect. ${ }^{5}$ Re-

\footnotetext{
${ }^{3}$ For this and all other experiments reported in this article, the same pattern of results was observed without this relative outlier exclusion.

${ }^{4}$ No by-item random slopes were included because in this case, the model did not reliably converge in the power analysis reported later.

${ }^{5}$ Models for which the congruency effect was modelled as an interaction between response direction and sentence direction did
} 


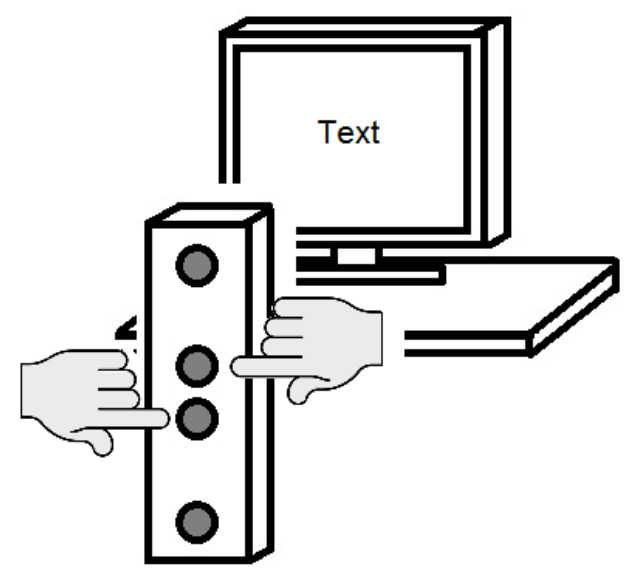

Figure 2. The experimental setup as seen from a participant's perspective. The overlay's buttons are placed over the $T A B, u, O$ and END keys of the keyboard.

lease times in the congruent condition $(M=1604 \mathrm{~ms})$ were faster than in the incongruent condition $(M=1648 \mathrm{~ms})$, amounting to a congruency effect of $44 \mathrm{~ms}$ (see Figure 3). ${ }^{6}$ Applying the method proposed by Westfall, Kenny, and Judd (2014) (see Brysbaert \& Stevens, 2018), this corresponds to a small effect size of $d=0.27$ in the mixed-effects model. For reference, we also conducted a supplemental $F_{1} / F_{2}$-ANOVA analysis (see Supplemental Material A), which indicates a medium effect size (partial $\eta_{F_{1}}^{2}=0.26$ and partial $\eta_{F_{2}}^{2}=.07$ ). ${ }^{7}$ As demonstrated by Brysbaert and Stevens (2018), the estimation of higher effect sizes in the $F_{1} / F_{2}$-approach is due to the fact that the data always enters this analysis in an aggregated format (by participant or by item), which results in far less variance than in the raw trial-based data that enters the mixed-effect model analysis (and the effect size $d$ by definition always compares the observed difference between conditions against the variance of the data). The congruency effect is not due to a speed-accuracy tradeoff: Participants made fewer errors in the congruent condition (percent correct: 88.7 $\%$ ) than in the incongruent condition (percent correct: 87.5 $\%)$.

In summary, the well-above chance performance demonstrates that people can learn and use the novel concepts. Furthermore, the results demonstrate that our experimental setting is suitable to detect congruency effects during tasks that require language comprehension, even when the actions described in the sentences include novel concepts.

\section{Experiment 2}

As stated in the Introduction, the critical second experiment investigates whether novel concepts learned from language alone can be indirectly grounded in sensorimotor experience. In this second experiment, the sentences involve the novel- word labels for these novel concepts, to rule out that the congruency effect observed in the first experiment is caused by the mere presence of vertically-related words (such as "head" or "foot") in the sentences.

\section{Method}

\section{Participants.}

Power Analysis. The sample size for Experiment 2 was determined through a power analysis using the reaction time data obtained in Experiment 1. Based on the mixedeffect model including the significant congruency fixed effect reported for Experiment 1, 1000 new data sets, each containing $n$ participants, were simulated using the lme 4 package's simulate() function (Bates et al., 2015). In these data, trials were randomly labelled as error trials based on the error percentage by congruency in Experiment 1, and then excluded from the data sets, to not over-estimate the amount of available data. No outlier analysis was applied in the simulations, since the model was already estimated on outlierremoved data. Then, a model including the same effect structure (fixed effect for congruency, by-participant and by-item

not converge, unless the random effect structure was reduced so far as to include no random slopes. We therefore opted for the more stable option of modelling a single two-level congruency effect.

${ }^{6}$ Although the aggregated data - especially by participants does not appear normally distributed in this graph, the residuals of the mixed-effects model are extremely close to a normal distribution in this and all the following analyses, justifying the application of linear models.

${ }^{7}$ Conventionally, a partial $\eta^{2}$ value of 0.01 is considered a small effect, 0.06 is considered a medium effect, and 0.14 is considered a large effect (Cohen, 1988) 


\section{Experiment 1}

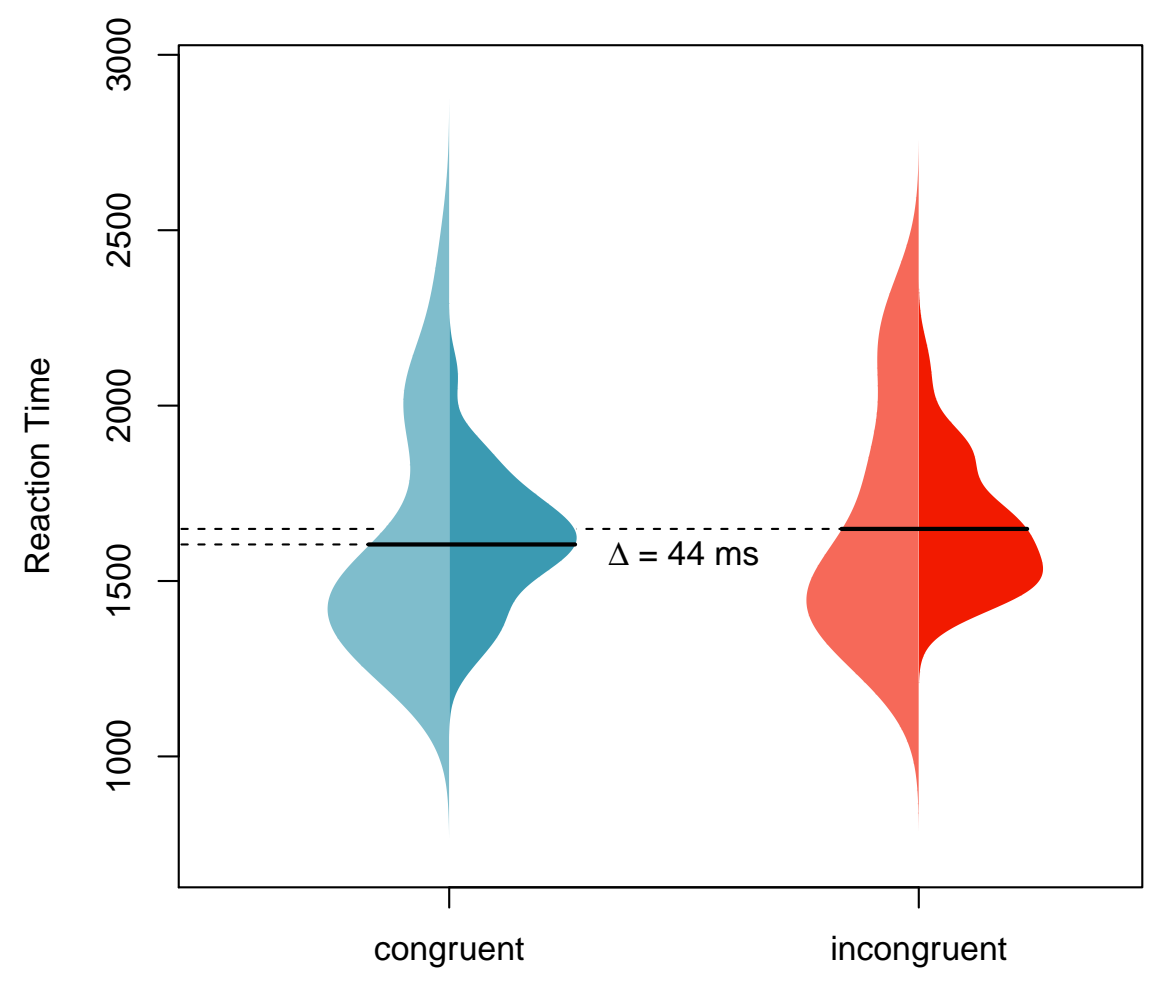

Condition

Figure 3. Reaction times (defined as release times) by condition in Experiment 1; distribution over participants (lighter left part) and items (darker right part). The solid lines indicate the mean values.

random intercepts, by-participant random slopes for congruency) was estimated for each of these new data sets. The percentage of models in which the congruency effect from which the data were generated was detected (i.e., for which $p<.05)$ was taken as the estimate for statistical power.

For $n=65$ simulated participants, we estimated a statistical power of .957 (i.e., in 957 out of 1000 simulation runs, the model detected a significant congruency effect). We opted for power estimates well above the .9 mark since it is reasonable to expect slightly smaller effects for the modified experimental design of Experiment 2, and since our power estimates were obtained from simulated data with less trimming (removal of error trials only) than the actual experimental data (where we remove error trials and RT-based outliers).

Participants. Since Experiment 2 is the critical experiment of this study, we wanted to ensure the robustness and replicability of our findings. We tested Experiment 2 in two separate runs, with two independent participant samples. None of the participants took part in Experiment 1.

The first sample included 68 participants (all native-
German speaking, 18 male, 53 female, $M_{\text {Age }}=21.9$ years, $S D_{\text {Age }}=3.0$ years). Seventeen additional participants were not included in the analysis due to high error rates (less than $70 \%$ correct for either sensible or nonsensical sentences). The second sample also included 68 participants (all nativeGerman speaking, 13 male, 55 female, $M_{\text {Age }}=23.3$ years, $S D_{\text {Age }}=7.8$ years). Here, 28 additional participants were not included in the analysis due to high error rates. The high exclusion rates reflect the greater difficulty of Experiment 2; however, participant exclusions do not affect the pattern of results reported here, as demonstrated in Supplemental Material A.

Material and Procedure. For the test phase in Experiment 2, we modified the sentence material used in Experiment 1 by replacing the novel concepts with the corresponding novel words that participants learned in the learning phase. Hence, if a participant learned that mende = enhanced head, she was presented with the sentence You scratch your mende instead of You scratch your enhanced 
head. Except for this modification, Experiment 2 was identical to Experiment 1. Due to the design of the item material, all sentences in Experiment 2 (such as You scratch your mende) can end up in any of the conditions, depending purely on the (randomized) learning phase (mende $=e n$ hanced head: upwards sensible; mende = bionic foot: downwards sensible; mende = grass carpet: implausible).

\section{Results}

Practice trials and nonsense items were excluded from the data set, as well as the data from participants with high error rates and high-error items $(<65 \%$ correct; two items in the first and three items in the second sample). In Experiment 2 , an item is defined at the meaning level of the original sentence (You scratch your enhanced head), irrespective of which novel word is used to describe the novel concept. Then, erroneous trials (13.8\% of the data in the first and 13.5 $\%$ in the second sample) were omitted from the data. We again excluded as absolute outliers trials with extremely high and extremely low reaction times (faster than the .1-quantile or slower than the .98-quantile; first sample: $<986 \mathrm{~ms}$ and $>$ $7324 \mathrm{~ms}$; second sample: < $834 \mathrm{~ms}$ and $>7490 \mathrm{~ms}$ ), and as relative outliers trials that deviated more than $2.5 S D$ s from the mean value per participant per condition (first sample: $2.1 \%$; second sample: $2.2 \%$ ).

We then fitted the same models as described for Experiment 1 and as used for the power analysis to test for a congruency effect (containing by-participant and byitem random intercepts and a by-participant random slope for congruency). In both participant samples, we observed a significant congruency effect, as the model including the congruency fixed effect significantly outperformed a model without this effect in a likelihood-ratio test (first sample: $\chi^{2}(1)=4.41, p=.036, \beta=0.02, t=2.14$; second sample: $\left.\chi^{2}(1)=9.40, p=.002, \beta=0.04, t=3.08\right)$. Release times in the congruent condition (first sample: $M=2511$ ms, second sample: $M=2367 \mathrm{~ms}$ ) were faster than in the incongruent condition (first sample: $M=2557 \mathrm{~ms}$, second sample: $M=2422 \mathrm{~ms}$ ), resulting in a congruency effect of $46 \mathrm{~ms}$ and $56 \mathrm{~ms}$, respectively (see Figure 4). In the mixedeffects models described here, this corresponds to small effect sizes: $d=0.14$ in the first sample and $d=0.15$ in the second sample (Brysbaert \& Stevens, 2018; Westfall et al., 2014). Again, we conducted supplemental $F_{1} / F_{2}$-ANOVAs for reference (see Supplemental Material A), which indicate medium effect sizes for both samples (first sample: partial $\eta_{F_{1}}^{2}=0.06$ and partial $\eta_{F_{2}}^{2}=.07$; second sample: partial $\eta_{F_{1}}^{2}$ $=0.13$ and partial $\eta_{F_{2}}^{2}=.13$ ). Note that release times in Experiment 2 were almost $1000 \mathrm{~ms}$ slower than in Experiment 1, again highlighting the greater difficulty of the task. Participants did not make more errors in the congruent condition (percent correct: first sample $86.2 \%$, second sample:
$87.8 \%$ ) than in the incongruent condition (percent correct: first sample $86.3 \%$, second sample: $85.1 \%$ ), indicating that the congruency effect is not the result of a speed-accuracy trade-off. In summary, the results from both runs indicate that novel words naming novel concepts can be grounded in sensorimotor experience.

\section{English Experiments}

In addition to the German experiments reported here, we performed the same two experiments in English. We observed a congruency effect of $38 \mathrm{~ms}$ in the English Experiment 1, but not in the English Experiment 2 (the congruency effect was non-significant at $11 \mathrm{~ms}$ ). However, we encountered serious problems with participant compliance and data quality in the English Experiment 2 (almost half of the tested participants had to be excluded due to not passing our error rate criterion), which render these results un-interpretable. We discuss these issues in Supplementary Material B, where both experiments are described in detail.

\section{General Discussion}

How do we represent novel concepts encountered solely through language? Traditional cognitive approaches suggest that such concepts derive their meaning from the web of associations amongst abstract symbols derived from linguistic co-occurrence (e.g., Landauer \& Dumais, 1997). The congruency effect observed in the present experiments significantly extends upon this view, by demonstrating that novel concepts and the meanings of novel words referring to them, upon initial learning, can be indirectly grounded in the sensorimotor system through this web of associations (see Snefjella \& Kuperman, 2016). That is, they are connected to sensorimotor experience, and this connection is established via familiar concepts that are already grounded.

\section{Experimental considerations}

Due to the design of the second experiment, a congruency effect observed there cannot occur because the nonwords are inherently associated with a vertical location (see the ratings of the material and experimental results in Günther et al., 2018), or because the used verbs inherently describe upwards- or downwards-actions: First, we selected only neutral verbs such as scratch, and more importantly, the same verbs were used to describe upwards- or downwards-actions, depending on the noun with which they were paired. Furthermore, since all verbs occurred in both sensible and nonsensical sentences, participants could not rely on the verb semantics to judge the sensibility of a sentence. Therefore, since the very same sentence (You scratch your mende) could end up in any condition depending purely on the learning phase, the congruency effect necessarily results from the novel word meaning acquired in the learning phase. 
Experiment 2 (first run)

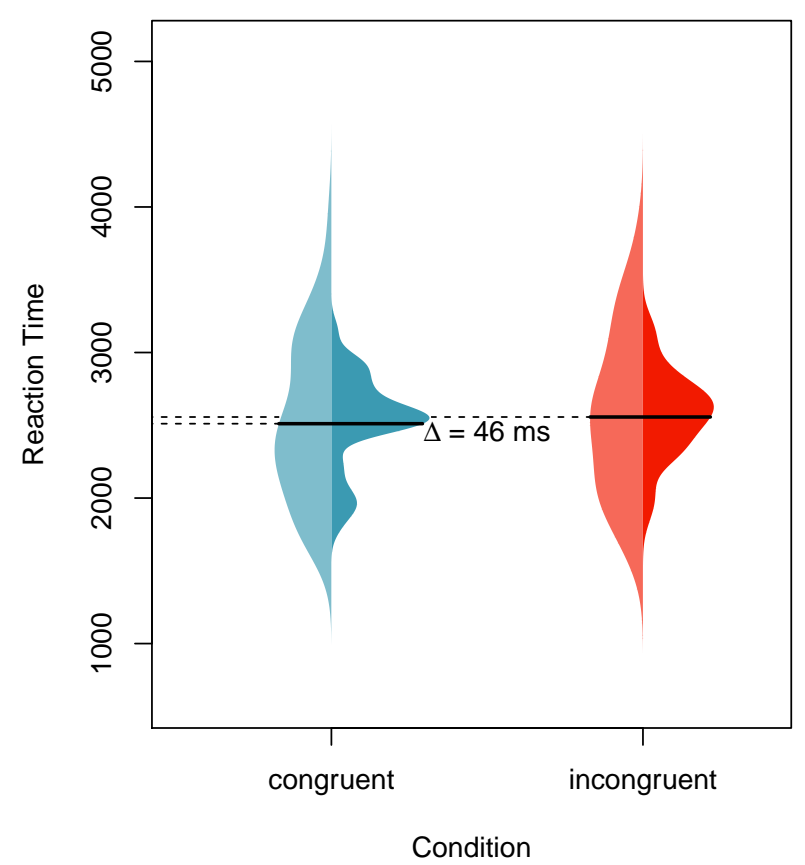

Experiment 2 (second run)

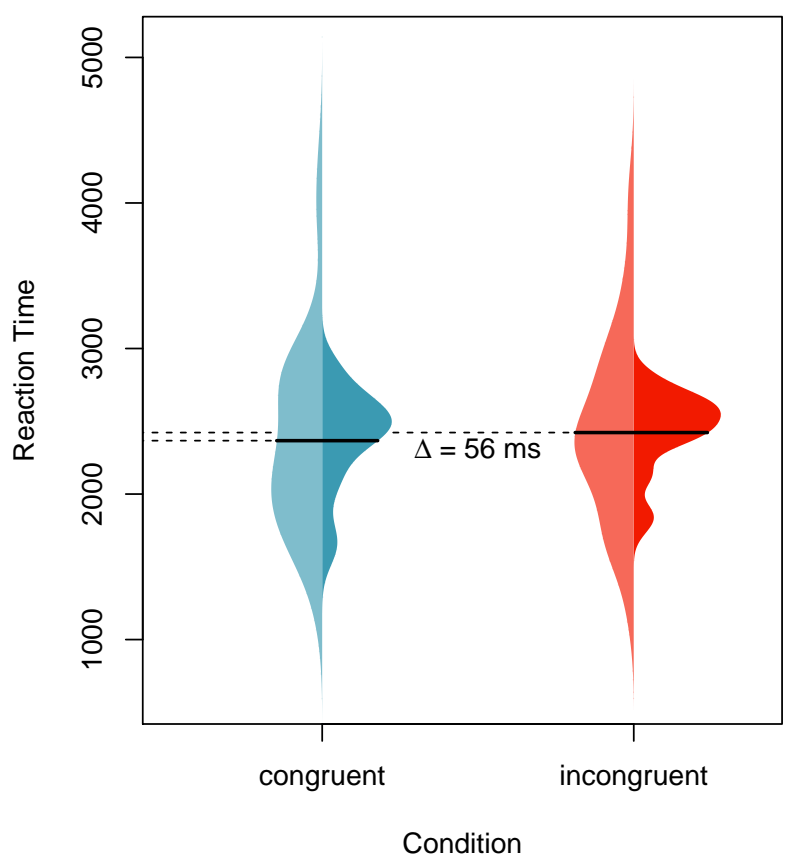

Figure 4. Reaction times (defined as release times) by condition in both runs of Experiment 2; distribution over participants (lighter left part) and items (darker right part). The solid lines indicate the mean values.

Of course, participants certainly have direct experience with the "un-modified" base concepts (such as foot or head). However, the congruency effect cannot be attributed to the participants just replacing the novel concepts with their respective familiar base concepts: Applying this strategy turns a sensible sentence into an implausible one for 10 out of the 40 items (e.g., You disassemble your [bionic] foot), thus leading to incorrect answers. However, the accuracies for these items are very similar to the ones where the replacement still results in a plausible sentence $(.87 \mathrm{vs} .89$ in Experiment 1, .85 vs .87 in the first run of Experiment 2, .84 vs .87 in the second run).

However, even if participants were to "internally substitute" the novel words, this would not speak against the assumption that indirect grounding is achieved via already grounded concepts (see Harnad, 1990). To judge the sensibility of the sentence "I rode home on a zebra", a valid strategy for our medieval European is to judge the sensibility of "I rode home on a striped horse". The substantially slower responses in our second experiment indeed suggest that such a substitution might indeed take place. However, the congruency effect in Experiment 2 also demonstrates that sensorimotor experience is activated while novel words learned from language alone are processed, which constitutes indirect grounding. Whether or not this indirect grounding in- volves the intermediary activation of the "base" concept (thus taking steps in the direction of a detailed processing model for indirectly-grounded concepts) is an empirical question for future studies.

Finally, a possible alternative explanation for congruency effects observed in the experimental paradigm employed here does not need to assume the involvement of sensorimotor activation: For example, in an fMRI study Francken, Kok, Hagoort, and De Lange (2015) observed compatibility effects for motion verbs only in brain areas associated to language processing, but not in areas associated to perceptual processing. Following these results, compatibility effects might also be explained as the result of high-level conceptual processing or decision making processes: This explanation would assume that both the linguistic input and the response movement are coded as "upward" or "downward" in the same disembodied, abstract format, with congruency effects resulting from matches or mismatches between these abstract codes. However, Dudschig and Kaup (2017) also observe motor compatibility effects when the mapping between the judgment and the response direction is randomly varied from trial to trial and indicated by a perceptual cue, which renders such an explanation less likely. In addition, referring to studies by Wallentin et al. (2011) and Saygin, McCullough, Alac, and Emmorey (2010), Francken et al. 
(2015) (p. 183) point out that "when the linguistic context is stronger, that is, when stimuli are sentences or narratives describing motion, studies have found activation of motion processing areas more proximal to MT+" (i.e., the perceptual system). This directly mirrors the discrepancy between the results by Günther et al. (2018), who observed no compatibility effects using single words and a superficial task, and the present results, where we observe compatibility effects in a stronger linguistic context, using sentence material and semantic judgments.

One can of course ask the general question if and to what extent behavioral experiments can answer questions regarding the embodiment of language processing, as any motor response most likely involves both sensorimotor and abstract code representations (Giesen \& Rothermund, 2016). Although empirical evidence indicates that interactions between linguistic input and motor responses (as observed in the present study) include sensorimotor activation and do not act solely on the abstract level (Aschersleben \& Prinz, 1997; Kunde, 2003), future research is required to fully disentangle these options (see Eder \& Rothermund, 2008, for exemplary studies on approach-avoidance behavior, or García et al., 2019, for possible neuropsychological approaches).

\section{Theoretical Implications}

On a theoretical level, our study demonstrates that novel concepts do not require direct sensorimotor experience to obtain a sensorimotor grounding. Instead, grounding can come about indirectly by establishing a connection via the alreadygrounded words defining the novel concept. In scenarios that require comprehension and thus semantic access to the conceptual system - for example, to understand the meaning of sentences - we observe congruency effects, which is in line with the view that processing involves the activation of sensorimotor experience. This is where the current study crucially deviates from previous studies implementing tasks that do not demand comprehension (Günther et al., 2018).

This grounding can be achieved by relying on the existing conceptual system and semantic representations, and extrapolating from there (Harnad, 1990). Here, we demonstrate that language serves as a central tool to establish this indirect grounding, and thus plays a vital role in scaling up our conceptual system. As put by Johnson-Laird (1983, p. 430), "a major function of language is thus to enable us to experience the world by proxy". This also highlights the important role played by the experience with linguistic stimuli in the model by Zwaan and Madden (2005): While most studies on grounded cognition do not explicitly focus on these linguistic traces, but mainly consider them only in their role as cues to re-activate sensorimotor experience, our present study shows that the interconnections between these traces can also play a vital link in the grounding of concepts and word meanings: They provide indirect routes and bridges to sensorimotor grounding.

This role of language as a means to establish indirect grounding of (novel) concepts has also been discussed with respect to the notion of semantic prosody, describing the phenomenon that specific words tend to occur in connotated contexts (see Winter, 2019) (for example, utterly is usually used in negative contexts). Moving from this, Snefjella and Kuperman (2016) found influences of the concreteness, valence, and arousal of their typical context words on the representation and processing of words, thus identifying the propagation of semantic properties through context as a general tendency in natural language. In a more recent study, Snefjella et al. (in press) further provide direct experimental evidence that originally meaningless novel words acquire emotional connotations from their learning context. This leads these authors to conclude that the "grounding of words might take place as a result of co-occurrence with words with sensorimotor or affective connotations" (Snefjella \& Kuperman, 2016, p. 144), which is in line with our initial hypothesis and the present results.

The role of sensorimotor experience in comprehension. The present study employed a task that requires reading for comprehension. In this task, we observed a congruency effect, which was not observed in earlier studies employing shallower paradigms that did not even require lexical access (Günther et al., 2018). Any sensorimotor experience would need to be activated during this task; otherwise, the participants' responses could not have been affected. However, as mentioned in the Introduction to this article, the actual role of this sensorimotor activation is highly debated in the literature (Barsalou, Simmons, Barbey, \& Wilson, 2003; Glenberg \& Kaschak, 2002; Goldinger, Papesh, Barnhart, Hansen, \& Hout, 2016; Mahon \& Caramazza, 2008; see Fischer \& Zwaan, 2008; Kaup, de la Vega, Strozyk, \& Dudschig, 2015, for overviews). To highlight this, let us consider the extreme sides in this debate.

According to some theoretical accounts (Barsalou et al., 2003; Glenberg, 2015; Glenberg \& Kaschak, 2002; Pulvermüller, Hauk, Nikulin, \& Ilmoniemi, 2005), the activation of sensorimotor activation plays a constitutive and causal role for comprehension: It is required and necessary for comprehension, or at least comprehension is impaired without it (Dreyer et al., 2015). Under this assumption, our medieval farmer would not be able to understand the sentence "I rode a zebra" without grounding the concept "zebra". In its final consequence, this view can assume that concepts are constituted by or simply identical to sensorimotor experience (Glenberg, 2015). According to this perspective, we observe a congruency effect in our experiments because a reader needs to simulate the actions described in the sentences (Glenberg \& Kaschak, 2002) - which include novel concepts - in order to understand them, and this simulation of action is necessarily based on sensorimotor experience. 
However, other theories assume the polar opposite (Goldinger et al., 2016; Mahon \& Caramazza, 2008), and postulate that sensorimotor activation is a pure epiphenomenon of comprehension: Like exhaust gas coming out of a car being a mere by-product of the actual process that makes the car run (fuel combustion in the engine), without any functionality itself, sensorimotor activation just occurs while comprehension is happening, but comprehension would not be impeded if it didn't happen (see Strozyk et al., 2019).

How do our results fit into that debate? The only logically warranted answer to this question is that they leave us agnostic, and our study was neither intended nor designed to answer this question. With respect to the causal-role view, we only demonstrate any sensorimotor experience would need to be activated during a task that requires comprehension. This does not imply that it is needed for comprehension. Therefore, our results leave open the possibility that it is just an epiphenomenon. However, even this latter interpretation would imply that sensorimotor activation occurs automatically, as it is in no way required to perform the task at hand: The sensibility of an action described in our sentence did not depend on the vertical location of the object.

The fact that reading a sentence such as "You scratch your mende" affects upwards or downwards movements in this semantic task - depending purely on which meaning was learned for mende - thus offers strong evidence for the activation of sensorimotor experience (keeping in mind the previous discussion of the possible alternative explanation that both language input as well as responses could be coded in an abstract format, as discussed earlier; Francken et al., 2015). This in turn requires this experience to be connected to the novel concepts. Given our minimalist definition of grounding, this directly translates to demonstrating that novel concepts and novel word meanings can be indirectly grounded in sensorimotor experience via language - the core research question of the present study. The question of causality has to be addressed in studies other than the present one, and a large body of such studies already exists (e.g. Dreyer et al., 2015; Mahon \& Caramazza, 2008; Strozyk et al., 2019).

A similar argument can be made concerning the time-course of sensorimotor activation (see Barsalou, Santos, Simmons, \& Wilson, 2008, for a time-course model). We have one piece of information backed by the empirical evidence of our studies: The existence of the congruency effect implies that any sensorimotor activation needs to occur at the point in time where the response is being made, or at least in sufficient temporal proximity to influence the response. This does not indicate whether it sets in before, during, or even after linguistic processing. It also doesn't indicate whether there still is linguistic activation when the response is being initiated or executed - in principle, linguistic processing and the resulting sensibility judgement can already be completed before the onset of sensorimotor activation sets in (Mahon \& Caramazza, 2008). It could even be the case that sensorimotor activation is not actually part of regular language processing, but just sets in because a motor response is required, or even just because a vertical motor response is required (see Dudschig \& Kaup, 2017).

The last scenario pushes sensorimotor activation furthest to a state of functional irrelevancy - a simple afterthought of processing (Mahon \& Caramazza, 2008). However, even in this case, we need to accommodate the finding that a motor response is affected by the semantic content of a preceding sentence. Thus, even in this scenario, our core argument holds: The novel concepts are connected to sensorimotor experience, which is evident by the fact that it can be activated when prompted - not even required - by a specific task. Of course, our study cannot confirm the claim that the entire conceptual system is grounded in sensorimotor experience. However, it does demonstrate that one major challenge - posed by novel concepts learned from language alone can be faced and successfully overcome. This is a major stepping stone in scaling up sensorimotor grounding to the non-experienced parts of the conceptual system.

\section{Conclusion}

In twenty years of research on grounded cognition, one of the most contested debates is and remains whether semantic representations are grounded in the sensorimotor system, and if so, to what extent (Meteyard, Cuadrado, Bahrami, \& Vigliocco, 2012). By remaining on this level of the debate, the field would be at risk of establishing a stalemate battleground without much theoretical progress in either direction (Ostarek \& Huettig, 2019). To overcome this stalemate, we argue that the objective has to be a thorough investigation how a theory can account for as many different cognitive phenomena as possible. This allows for a broader appreciation and evaluation of the actual predictive power of a theoretical account.

With the present article, we thus set out to contribute to re-framing the debate by moving away from a yesor-no focus. Instead, we build a grounded account of a central cognitive phenomenon - the expansion of the conceptual system - and test its limits (Ostarek \& Huettig, 2019). We demonstrate how our conceptual system scales up beyond limited direct sensorimotor experience (Landauer \& Dumais, 1997) by utilizing two of our most powerful cognitive tools: language and grounded simulation.

\section{References}

Aschersleben, G., \& Prinz, W. (1997). Delayed auditory feedback in synchronization. Journal of Motor Behavior, 29, 35-46. 
Baayen, R. H., Davidson, D. J., \& Bates, D. M. (2008). Mixedeffects modeling with crossed random effects for subjects and items. Journal of Memory and Language, 59, 390-412.

Baayen, R. H., \& Milin, P. (2010). Analyzing reaction times. International Journal of Psychological Research, 3(2), 12-28.

Barr, D. J., Levy, R., Scheepers, C., \& Tily, H. J. (2013). Random effects structure for confirmatory hypothesis testing: Keep it maximal. Journal of Memory and Language, 68, 255-278.

Barsalou, L. W. (1999). Perceptual symbol systems. Behavioral and Brain Sciences, 22, 637-660.

Barsalou, L. W. (2008). Grounded cognition. Annual Review of Psychology, 59, 617-645.

Barsalou, L. W., Santos, A., Simmons, W. K., \& Wilson, C. D. (2008). Language and simulations in conceptual processing. In M. D. Vega, A. M. Glenberg, \& A. C. Graesser (Eds.), Symbols and embodiment: Debates on meaning and cognition (pp. 245283). Oxford, UK: Oxford University Press.

Barsalou, L. W., Simmons, W. K., Barbey, A. K., \& Wilson, C. D. (2003). Grounding conceptual knowledge in modality-specific systems. Trends in Cognitive Sciences, 7, 84-91.

Bates, D., Mächler, M., Bolker, B., \& Walker, S. (2015). Fitting linear mixed-effects models using lme4. Journal of Statistical Software, 67(1), 1-48.

Borghi, A. M., \& Binkofski, F. (2014). Words as social tools: An embodied view on abstract concepts. Berlin, Germany, and New York, NY: Springer. doi: 10.1007/978-1-4614-9539-0

Borghi, A. M., Binkofski, F., Castelfranchi, C., Cimatti, F., Scorolli, C., \& Tummolini, L. (2017). The challenge of abstract concepts. Psychological Bulletin, 143, 263-292.

Borghi, A. M., Glenberg, A. M., \& Kaschak, M. P. (2004). Putting words in perspective. Memory $\mathcal{E}$ Cognition, 32, 863-873.

Brysbaert, M., \& Stevens, M. (2018). Power analysis and effect size in mixed effects models: A tutorial. Journal of Cognition, 1(1), 9.

Brysbaert, M., Stevens, M., Mandera, P., \& Keuleers, E. (2016). How many words do we know? practical estimates of vocabulary size dependent on word definition, the degree of language input and the participant's age. Frontiers in Psychology, 7, 1116.

Clark, H. H. (1973). The language-as-fixed-effect fallacy: A critique of language statistics in psychological research. Journal of verbal learning and verbal behavior, 12, 335-359.

Cohen, J. (1988). Statistical Power Analysis for the Behavioral Sciences (2nd ed.). Hillsdale, NJ: Erlbaum.

Dreyer, F. R., Frey, D., Arana, S., Saldern, S. v., Picht, T., Vajkoczy, P., \& Pulvermüller, F. (2015). Is the motor system necessary for processing action and abstract emotion words? Evidence from focal brain lesions. Frontiers in Psychology, 6, 1661.

Dudschig, C., \& Kaup, B. (2017). Is it all task-specific? The role of binary responses, verbal mediation, and saliency for eliciting language-space associations. Journal of Experimental Psychology: Learning, Memory, and Cognition, 43, 259-270.

Eder, A. B., \& Rothermund, K. (2008). When do motor behaviors (mis)match affective stimuli? an evaluative coding view of approach and avoidance reactions. Journal of Experimental Psychology: General, 137, 262-281.

Fischer, M. H. (2012). A hierarchical view of grounded, embodied, and situated numerical cognition. Cognitive Processing, 13,
$161-164$

Fischer, M. H., \& Zwaan, R. A. (2008). Embodied language: A review of the role of the motor system in language comprehension. The Quarterly Journal of Experimental Psychology, 61, $825-850$.

Francken, J. C., Kok, P., Hagoort, P., \& De Lange, F. P. (2015). The behavioral and neural effects of language on motion perception. Journal of Cognitive Neuroscience, 27, 175-184.

García, A. M., Moguilner, S., Torquati, K., García-Marco, E., Herrera, E., Muñoz, E., ... Ibáñez, A. (2019). How meaning unfolds in neural time: Embodied reactivations can precede multimodal semantic effects during language processing. NeuroImage, 197, 439-449.

Giesen, C., \& Rothermund, K. (2016). Multi-level response coding in stimulus-response bindings: Irrelevant distractors retrieve both semantic and motor response codes. Journal of Experimental Psychology: Learning, Memory, and Cognition, 42, 16431656.

Glenberg, A. M. (2015). Few believe the world is flat: How embodiment is changing the scientific understanding of cognition. Canadian Journal of Experimental Psychology, 69, 165-171.

Glenberg, A. M., \& Kaschak, M. P. (2002). Grounding language in action. Psychonomic Bulletin $\mathcal{F}$ Review, 9, 558-565.

Goldinger, S. D., Papesh, M. H., Barnhart, A. S., Hansen, W. A., \& Hout, M. C. (2016). The poverty of embodied cognition. Psychonomic Bulletin $\mathcal{E}$ Review, 23, 959-978.

Günther, F., Dudschig, C., \& Kaup, B. (2018). Symbol grounding without direct experience: Do words inherit sensorimotor activation from purely linguistic context? Cognitive Science, 42, 336-374.

Günther, F., Rinaldi, L., \& Marelli, M. (2019). Vector-space models of semantic representation from a cognitive perspective: A discussion of common misconceptions. Perspectives on Psychological Science, 14, 1006-1033.

Harnad, S. (1990). The symbol grounding problem. Physica D: Nonlinear Phenomena, 42, 335-346.

Johnson-Laird, P. N. (1983). Mental Models: Towards a Cognitive Science of Language, Inference, and Consciousness. Cambridge, MA: Harvard University Press.

Kaup, B., de la Vega, I., Strozyk, J., \& Dudschig, C. (2015). The role of sensorimotor processes in meaning composition. In M. H. Fischer \& Y. Coello (Eds.), Conceptual and interactive embodiment (pp. 58-82). London, UK: Routledge.

Kousta, S.-T., Vigliocco, G., Vinson, D. P., Andrews, M., \& Del Campo, E. (2011). The representation of abstract words: Why emotion matters. Journal of Experimental Psychology: General, 140, 14-34.

Kunde, W. (2003). Temporal response-effect compatibility. Psychological Research, 67(3), 153-159.

Kuznetsova, A., Brockhoff, P. B., \& Christensen, R. H. B. (2017). lmerTest package: Tests in linear mixed effects models. Journal of Statistical Software, 82(13), 1-26.

Lachmair, M., Dudschig, C., De Filippis, M., de la Vega, I., \& Kaup, B. (2011). Root versus roof: automatic activation of location information during word processing. Psychonomic Bulletin $\mathcal{E}$ Review, 18, 1180-1188.

Lakoff, G., \& Johnson, M. (1980). The metaphorical structure of the human conceptual system. Cognitive Science, 4, 195-208. 
Landauer, T. K., \& Dumais, S. T. (1997). A solution to Plato's problem: The Latent Semantic Analysis theory of acquisition, induction, and representation of knowledge. Psychological Review, 104, 211-240.

Lawrence, M. A. (2016). ez: Easy Analysis and Visualization of Factorial Experiments [Computer software manual]. Retrieved from https : //CRAN.R-project . org/package=ez (R package version 4.4-0)

Lebois, L. A., Wilson-Mendenhall, C. D., \& Barsalou, L. W. (2015). Are automatic conceptual cores the gold standard of semantic processing? The context-dependence of spatial meaning in grounded congruency effects. Cognitive Science, 39, 17641801.

Mackenzie, I. G. (2019). psychReport: Reproducible Reports in Psychology [Computer software manual]. Retrieved from https: //CRAN . R-project . org/package=psychReport (R package version 0.7 )

Mahon, B. Z. (2015). The burden of embodied cognition. Canadian Journal of Experimental Psychology, 69, 172-178.

Mahon, B. Z., \& Caramazza, A. (2008). A critical look at the embodied cognition hypothesis and a new proposal for grounding conceptual content. Journal of Physiology-Paris, 102(1-3), 59-70.

Meteyard, L., Cuadrado, S. R., Bahrami, B., \& Vigliocco, G. (2012). Coming of age: A review of embodiment and the neuroscience of semantics. Cortex, 48, 788-804.

Ostarek, M., \& Huettig, F. (2019). Six challenges for embodiment research. Current Directions in Psychological Science, Advance online publication. doi: 10.1177/0963721419866441

Öttl, B., Dudschig, C., \& Kaup, B. (2017). Forming associations between language and sensorimotor traces during novel word learning. Language and Cognition, 9, 156-171.

Papesh, M. H. (2015). Just out of reach: On the reliability of the action-sentence compatibility effect. Journal of Experimental Psychology: General, 144, e116-e141.

Pecher, D. (2018). Curb your embodiment. Topics in Cognitive Science, 10, 501-517.

Pulvermüller, F., Hauk, O., Nikulin, V. V., \& Ilmoniemi, R. J. (2005). Functional links between motor and language systems. European Journal of Neuroscience, 21, 793-797.

Rights, J. D., \& Sterba, S. K. (2019). Quantifying explained variance in multilevel models: An integrative framework for defining R-squared measures. Psychological Methods, 24, 309.

Saygin, A. P., McCullough, S., Alac, M., \& Emmorey, K. (2010). Modulation of bold response in motion-sensitive lateral temporal cortex by real and fictive motion sentences. Journal of Cognitive Neuroscience, 22, 2480-2490.

Snefjella, B., \& Kuperman, V. (2016). It's all in the delivery: Effects of context valence, arousal, and concreteness on visual word processing. Cognition, 156, 135-146.

Snefjella, B., Lana, N., \& Kuperman, V. (in press). How emotion is learned: Semantic learning of novel words in emotional contexts. Journal of Memory and Language.

Strozyk, J. V., Dudschig, C., \& Kaup, B. (2019). Do I need to have my hands free to understand hand-related language? Investigating the functional relevance of experiential simulations. Psychological Research, 83, 406-418.

Thornton, T., Loetscher, T., Yates, M. J., \& Nicholls, M. E. (2013).
The highs and lows of the interaction between word meaning and space. Journal of Experimental Psychology: Human Perception and Performance, 39, 964-973.

Vigliocco, G., Kousta, S.-T., Della Rosa, P. A., Vinson, D. P., Tettamanti, M., Devlin, J. T., \& Cappa, S. F. (2013). The neural representation of abstract words: the role of emotion. Cerebral Cortex, 24, 1767-1777.

Wallentin, M., Nielsen, A. H., Vuust, P., Dohn, A., Roepstorff, A., $\&$ Lund, T. E. (2011). Bold response to motion verbs in left posterior middle temporal gyrus during story comprehension. Brain and Language, 119, 221-225.

Westfall, J., Kenny, D. A., \& Judd, C. M. (2014). Statistical power and optimal design in experiments in which samples of participants respond to samples of stimuli. Journal of Experimental Psychology: General, 143, 2020.

Winter, B. (2019). Sensory linguistics: Language, perception and metaphor (converging evidence in language and communication research, vol. 20). Amterdam, The Netherlands: John Benjamins Publishing Company.

Zwaan, R. A., \& Madden, C. J. (2005). Embodies sentence comprehension. In D. Pecher \& R. A. Zwaan (Eds.), Grounding cognition: The role of action and perception in memory, language, and thinking (pp. 224-245). Cambridge, UK: Cambridge University Press. 


\section{Supplementary Material A:}

\section{Analysis 1: $F_{1} / F_{2}$-ANOVAs and effect size estimates}

In the main manuscript, we report analyses based on Linear Mixed Effect Models (LMEMs). For our data structure, these analysis are more adequate than the more traditional $F_{1} / F_{2}$-ANOVA approach, for example because they consider random variation due to participants and items simultaneously, and because the analysis can run on the raw trialbased data instead of aggregated data. As a consequence, the LMEM approach has been demonstrated to be superior than the $F_{1} / F_{2}$-ANOVA approach, for example with respect to controlling for Type I errors (Baayen et al., 2008; Barr, Levy, Scheepers, \& Tily, 2013). However, there currently is no consensus on how to compute effect sizes in the LMEM approach (Rights \& Sterba, 2019).

In order to still provide some indication on effect sizes in our studies, we supplement our main analysis with a more traditional but demonstrably sub-optimal $F_{1} / F_{2-}$ ANOVA analysis (i.e., one within-subject ANOVA, and one within-items ANOVA Clark, 1973). The analyses were conducted using the R package $e z$ (Lawrence, 2016), and effect sizes (partial $\eta^{2}$ ) were estimated using the package $p s y c h R e$ port (Mackenzie, 2019). Conventionally, a partial $\eta^{2}$ value of 0.01 is considered a small effect, 0.06 is considered a medium effect, and 0.14 is considered a large effect (Cohen, 1988).

Applying the same data-cleaning procedures as described in the main article, the results are as follows: In Study 1 , the congruency effect is significant in the by-participant analysis $\left(F_{1}(1,48)=16.58, p<.001\right.$, partial $\eta^{2}=0.257$; $F_{2}(1,37)=2.70, p=.109$, partial $\left.\eta^{2}=0.068\right)$. We observe the same pattern for the first run of Study $2\left(F_{1}(1,67)=4.27\right.$, $p=.043$, partial $\eta^{2}=0.060 ; F_{2}(1,37)=2.74, p=.106$, partial $\left.\eta^{2}=0.069\right)$. In the second run of Study 2 , the congruency effect is significant in both analyses $\left(F_{1}(1,67)=10.33\right.$, $p=.002$, partial $\eta^{2}=0.134 ; F_{2}(1,36)=5.41, p=.003$, partial $\eta^{2}=0.131$ ). Thus, across all studies, the $F_{1} / F_{2}$ ANOVAs suggest at effect sizes that would conventionally be considered as medium.

\section{Analysis 2: The main analyses without participant exclu- sion}

Especially in the two runs of Study 2, we excluded a considerable portion of participants due to their high error rates. This might however introduce some selection bias in our study, which might affect the results. We therefore replicated the same analyses without excluding any participants. All trial-based exclusion criteria however, including exclusions of error trials, were maintained.

In Study 1 (where we only excluded one participant in the first place), the pattern of results did not change: An LMEM that includes a congruency effect outperforms an LMEM without this effect $\left(\chi^{2}(1)=10.63, p=.001\right.$, $b=0.03, t=3.27)$. The same is true for the first run of Study 2 , when based on all 85 original participants $\left(\chi^{2}(1)=4.94\right.$, $p=.026, b=0.03, t=2.26$ ), as well as for the second run of Study 2, when based on all 96 original participants $\left(\chi^{2}(1)=9.66, p=.002, b=0.03, t=3.12\right)$. Thus, the pattern of results reported in the main analysis is not affected by the exclusion of participants. 


\section{Supplementary Material B: English Experiments}

\section{English Experiment 1}

\section{Method.}

Participants. In the English version of Experiment 1, we tested 107 native speakers of American English (53 identified as male, 46 as female, and 8 as other, $M_{\text {Age }}=19.4$ years, $S D_{\text {Age }}=3.5$ years). Four additional participants were not included in the analysis due to a high error rate, as described in more detail in the Results section of Experiment 1 in the main article. Participants gave informed consent to participating in the experiment, and received either money or course credit for their participation.

Material and Procedure. The material for the English version of Experiment 1 was adapted from the German version. All learning phase descriptions were translated by the authors, as were the test phase sentences. It was taken care that a one-to-one mapping between the verbs used in these test phase sentences was established (so the same verb in German always translated to the same verb in English).

Since the novel words in the German experiments were designed to be German-sounding, we constructed eight different English-sounding pseudowords for the English version (glaff, lourer, segmore, turry, ammer, billit, brince, caris). In a rating study, 34 participants judged whether they associated the pseudo-words with an upper or lower vertical location. None of these eight pseudo-words was significantly associated to a vertical location (proportion of up-ratings between .44 and .53).

Apart form these changes in language, the material and procedure of the English version was identical to the German version.

Results. Practice trials and nonsense items were excluded from the data set. Applying the same criteria as for the German experiments, we further eliminated the data from four participants with high error rates $(<70 \%$ correct for either sensible or nonsensical items) and two high-error items (correct answers not significantly above chance level, $<65 \%$ correct). Then, all error trials ( $12.9 \%$ of trials) were omitted from the data. We excluded as absolute outliers trials with extremely high and extremely low overall reaction times (faster than the .01-quantile, $782 \mathrm{~ms}$, or slower than the .98 -quantile, $4690 \mathrm{~ms}$ ), and as relative outliers trials that deviated more than 2.5 SDs from the mean value per participant per condition ( $2.1 \%$ of trials).

We again estimated a Linear Mixed Effects Model to predict logarithmic release times that included a fixed effect for congruency, random intercepts for participants and items, and by-participant random slopes for congruency.

The model including the congruency fixed effect significantly outperformed a model without this effect in a likelihood-ratio test $\left(\chi^{2}(1)=7.05, p=.008, b=0.02\right.$, $t=2.70)$, indicating a significant congruency effect. Release times in the congruent condition $(M=1686 \mathrm{~ms})$ were faster than in the incongruent condition $(M=1725 \mathrm{~ms})$, amounting to a congruency effect of $39 \mathrm{~ms}$ (see Figure 5). Participants made fewer errors in the congruent condition (percent correct: $88.9 \%$ ) than in the incongruent condition (percent correct: $87.5 \%$ ), indicating that there was no speed-accuracy trade-off.

Note that, in many respects, the results of this first experiment in English are quite comparable to its German counterpart: The number of participants, items, and trials excluded (both error trials and reaction time outliers) are very similar between the two. The overall reaction times are about $80 \mathrm{~ms}$ slower in the English experiment, and the numerical difference between the two conditions (i.e., the congruency effect) is somewhat smaller ( $39 \mathrm{~ms}$ as compared to $44 \mathrm{~ms}$ ), which is reflected in the $t$-values for the effect in the statistical models $(t=2.70$ in English as compared to $t=3.23$ in German).

\section{English Experiment 2}

\section{Method.}

Participants.

Power Analysis. We performed the same power analysis as reported for the German study (using the very same script), based on the English data in Experiment 1. The results of this analysis however differed strongly between the two languages: In order to reach a power of .9, the English experiment would require a sample size of over 160 participants. With 110 participants, the analysis still estimates a relatively high power level power of .78 .

Participants. For the English version of Experiment 2, we tested 106 participants. However, due to high error rates (less than $70 \%$ correct for either sensible or nonsensical sentences), we had to exclude 41 of these participants, only leaving us with a sample of 65 datasets to be analysed. This exclusion rate is dramatically higher than for both runs of the German version of Experiment 2. These 65 participants were English native speakers (39 male, 26 female, $M_{\text {Age }}=18.94$ years, $S D_{\text {Age }}=1.4$ years). Participants gave informed consent to participating in the experiment, and received either money or course credit for their participation.

Material and Procedure. Except for the change in language (see English Experiment 1), the English version of Experiment 2 was identical to the German version.

Results. Practice trials and nonsense items were excluded from the data set, as well as high-error items $(<65$ $\%$ correct; three items). Note that, as in the German version, "item" in Experiment 2 is defined at the meaning level. Then, erroneous trials (16.9\% of the data) were omitted. 


\section{English Experiment 1}

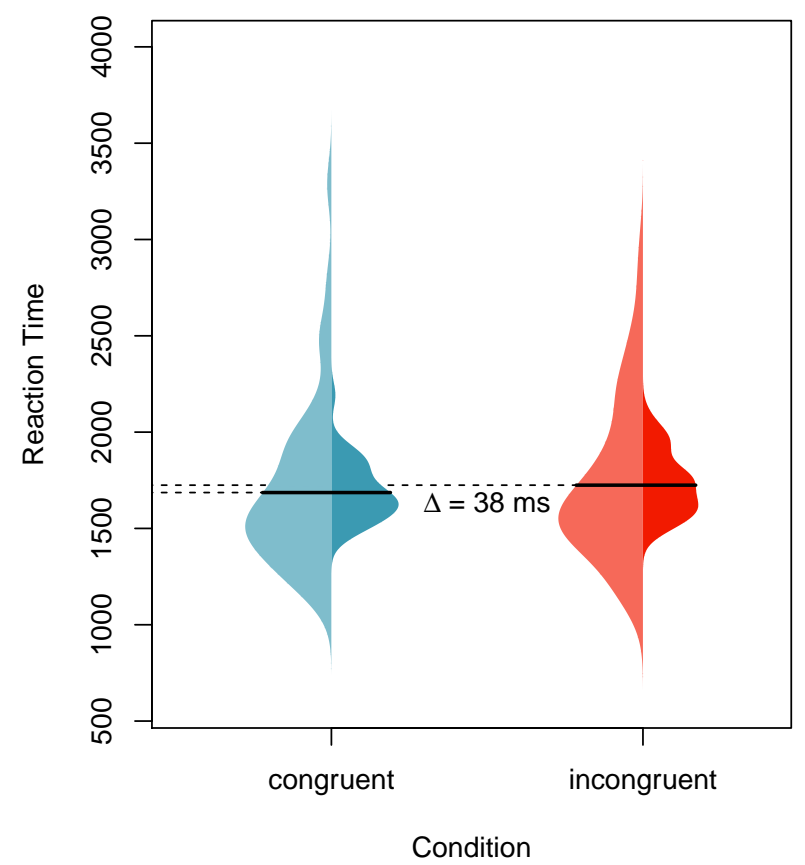

English Experiment 2

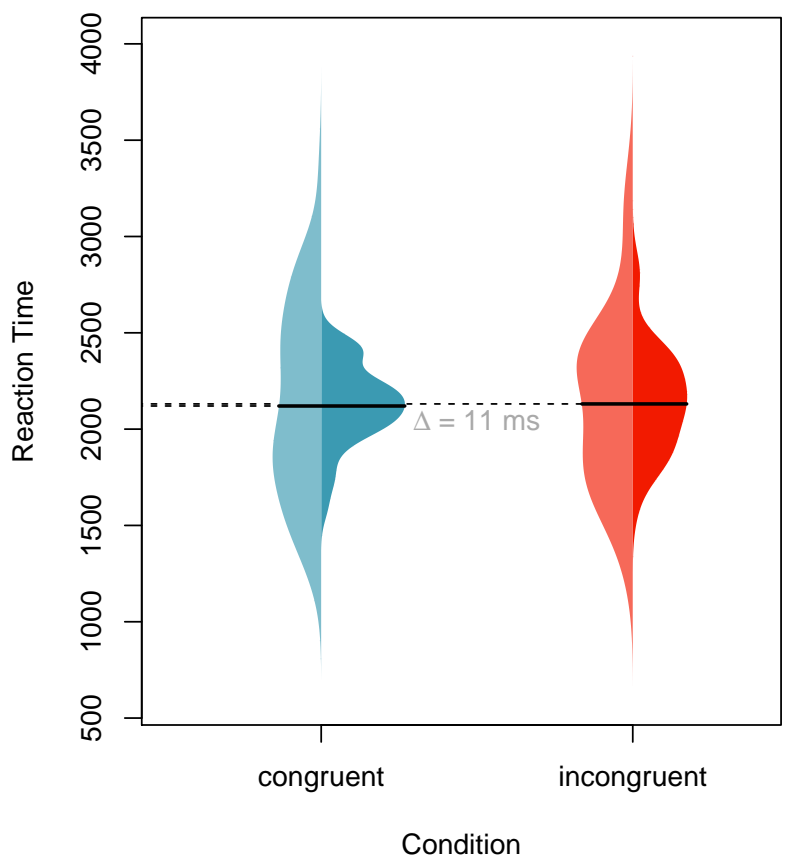

Figure 5. Reaction times (defined as release times) by condition in the English version of Experiment 1 (left) and Experiment 2 (right); distribution over participants (lighter left part) and items (darker right part). The solid lines indicate the mean values.

Thus, even after exclusion of high-error participants, we had to exclude more error trials in the remaining data than in the two runs of the German version $(13.8 \%$ and $13.5 \%$, respectively). We again excluded as absolute outliers trials with extremely high and extremely low reaction times (faster than the .1-quantile, $877 \mathrm{~ms}$ or slower than the .98 -quantile, $6149 \mathrm{~ms}$ ), and as relative outliers trials that deviated more than $2.5 S D$ s from the mean value per participant per condition (2.0\%, comparable to the German version).

In the Linear Mixed Effect Model analysis, we did not observe a significant congruency effect: The model including the congruency fixed effect did not outperform a model without this effect in a likelihood-ratio test $\left(\chi^{2}(1)=\right.$ $0.40, p=.525, \beta=0.01, t=0.64)$. On a numerical level, release times in the congruent condition $(M=2120 \mathrm{~ms})$ were smaller than in the incongruent condition $(M=2130 \mathrm{~ms})$. Note that, even though responses in the English version of Experiment 1 were slower than in the German version, the reaction times in Experiment 2 are much faster in the English version (around $400 \mathrm{~ms}$ faster than the first run of the German version of Experiment 2, and around $275 \mathrm{~ms}$ faster than the second run). Participants made fewer errors in the congruent condition (percent correct: $83.8 \%$ ) than in the incongruent condition (percent correct: $82.4 \%$ ).

\section{Discussion}

We observe a congruency in the English version of Experiment 1, but not in the English version of Experiment 2. However, we encountered serious problems in the data collection of Experiment 2:

We initially aimed to collect data from enough participants to reach a power level of .8 (note that the 110 participants required for this are already considerably more than for the German version). After finishing this data collection, we realized that more than $40 \%$ of participants had to be excluded; that is, almost half of the participants' performance was essentially not significantly different from chance level. For these participants, we cannot ensure that they actually remembered the word meanings acquired in the learning phase, or if they did, whether they tried to perform the test phase as instructed.

By excluding such a large percentage of participants, our dataset is highly biased. Furthermore, these participants are much faster than the German participants in Experiment 2 - even though participants in the English version of Experiment 1 were slightly slower - which again suggests a bias. We therefore decided to stop the data collection at this point. That is, although we could have collected more data to reach the sample size suggested by the power analysis (110), the data would be so biased as to be uninterpretable. Due to 
the resulting low statistical power of this experiment (with 65 participants, the power estimate is .51), the null effect in this experiment cannot be reliably interpreted in any direction. However, given that the immediate grounding of novel concepts in sensorimotor simulations is observed in two highpowered runs of Experiment 2 in German, the empirical evidence speaks in favour of the presence of such an effect.

This leaves the question of why the performance of English-speaking sample in Experiment 2 was out of line, as compared to the other experiments. Of course, we can only speculate at this point. We did not expect any principled differences between the languages themselves, and thus assume the difference to lie at the level of the participant sample. One possible point is that the German-speaking sample is essentially multilingual, since the German school curriculum includes at least one (and for most students multiple) foreign languages, which is not the case to the same extent in the US. This could be examined by identifying and testing a sample of German monolinguals, or US bilinguals. We can also not rule out the possibility that there are differences in motivation when participating in an experiment and overall effort and compliance between the two samples.

Finally, and related, the University of Tübingen (where we tested the German-speaking participants) is highly selective in the admission of students. In contrast, Arizona State University (where we tested the English-speaking participants) has a policy of admitting all students who meet basic qualifications. According the University's charter, the University is "measured not by whom it excludes, but by whom it includes...". The relatively unselected ASU students may have been less motivated than the highly-selected Tübingen students, especially given the relatively high difficulty of the task in Experiment 2. This issue could be investigated in future studies by replicating the experiment with an US sample from a highly competitive learning environment. 